methods for localizing STN during DBS surgery include the use of stereotactic coordinates and multi-electrode recording (MER) during implantation. MERs are incredibly useful in DBS surgeries, but require penetration of brain structures in order to infer location. Using multi-photon microscopy techniques to aid identification of STN during DBS surgeries offers a number of advantages over traditional methods. For example, blood vessels can be clearly identified with second harmonic generation, something that is not possible with MER. Multi-photon microscopy also allows visualization deep into tissue without actually penetrating it. This ability to look within a depth of field is useful for detection of STN borders based on autofluorescent cell density. When combined with traditional stereotactic information, our preliminary image classification methods are a fast, reliable way to provide surgeons with extra information concerning their location in the midbrain. We anticipate that future advancements and refinements to our image classifier will only increase accuracy and the potential applications and value. In summary, these preliminary data support the feasibility of multi-photon microscopy to aid in the identification of target brain regions during DBS surgeries. The techniques described here complement and enhance current stereotactic and electrophysiological methods for DBS surgeries.

A robust spatial normalization pipeline for individuals with focal cortical lesions

Andrew DeMarco and Peter Turkeltaub

Georgetown - Howard Universities

OBJECTIVES/SPECIFIC AIMS: To develop a robust spatial normalization pipeline for brains of individuals with focal cortical lesions. METHODS/STUDY POPULATION: Individuals with chronic focal cortical lesions from stroke. RESULTS/ ANTICIPATED RESULTS: We have developed a robust spatial normalization pipeline for brains of individuals with focal cortical lesions, and demonstrate how the pipeline overcomes obfuscated neuroanatomy to yield both consistent and excellent results. DISCUSSION/SIGNIFICANCE OF IMPACT: Our robust normalization pipeline will enable group analyses of individuals with cortical lesions with greater spatial precision. This greater spatial precision will improve answers to questions about functional localization in the brain, and ultimately allow translation of findings from neuroimaging studies in individuals with cortical lesions to the clinic.

\section{A systematic overview of cost-utility analyses in dermatology}

David G. Li', Adam Faletsky ${ }^{2}$, Peter Neumann ${ }^{2}$ and Joshua Cohen ${ }^{2}$

' School of Medicine, Tufts University; ${ }^{2}$ Tufts University

OBJECTIVES/SPECIFIC AIMS: Costs associated with the treatment of skin diseases accounted for greater than $4 \%$ of total US healthcare spending in 2013 , an increase of $\$ 46$ billion (170\%) since 2004 . Considering the increase in novel treatments and spending, cost-utility analyses (CUAs) may provide a better understanding of costs in dermatology. In this study, we conduct a systematic overview of study quality among CUAs related to dermatology. METHODS/STUDY POPULATION: We queried studies from the Tufts Medical Center Cost-Effectiveness Analysis Registry (www.cearegistry.org), a database supplying information on all peer-reviewed costeffectiveness analysis through 2014. Database methodology was previously discussed here. We queried studies using keywords from the 24 major skin disease categories (e.g., diseases relating to actinic damage were searched by using "actinic," "actinic keratosis"). We collected data on study design, reporting methods, and analyzed relevant data stratified by 2 time-periods (I976-2008 and 2009-2014) chosen to encompass a comparable number of studies. RESULTS/ANTICIPATED RESULTS: In total, 42 and 50 studies corresponding to the 2 time-periods were retrieved (representing 14/24 disease categories). Based on the recommended data reporting guidelines for CUAs, study quality remained largely unchanged across the 2 phases. Across the 2 time-periods, a societal perspective was used in $19 \%$ and $12 \%$ of studies, costs and (quality adjusted life-years) QALYs were discounted in $67 \%$ and $72 \%$ of studies, a correct (incremental cost-effectiveness ratio) ICER was reported in $67 \%$ and $72 \%$ of studies, and a sensitivity analysis was included in $88 \%$ and $84 \%$ of studies, respectively. DISCUSSION/SIGNIFICANCE OF IMPACT: Our findings suggest the quality of dermatology-related CUAs, as evaluated by recommended data reporting guidelines, to be generally stable during the analyzed time-periods. However, the quality of our results may be limited by the small number of CUAs within dermatology (10/24 disease categories did not have CUAs across any timeperiod). Moving forward, we encourage researchers within dermatology to pursue additional investigation towards cost-effective practices while adhering closely to recommended quality reporting guidelines for CUAs.
Acellular hyaluronic acid scaffold with growth factor delivery for cartilage repair in a large animal model Anthony R. Martín', Jay M. Patel ${ }^{2}$, Hannah M. Zlotnick ${ }^{2}$, Mackenzie L. Sennet ${ }^{3}$, James L. Carey ${ }^{2}$ and Robert L. Mauck ${ }^{3}$

' University of Pennsylvania School of Medicine, Philadelphia, PA, USA; ${ }^{2}$ University of Pennsylvania, Philadelphia, PA, USA;

${ }^{3}$ Translational Musculoskeletal Research Center, Medical Center, University of Pennsylvania, Philadelphia, PA, USA

OBJECTIVES/SPECIFIC AIMS: Focal cartilage injuries of the knee joint are common and present a treatment challenge due to minimal intrinsic repair. Cartilage tissue engineering techniques currently used in clinical practice are expensive, cumbersome, and often ineffective in patients with mechanical or medical comorbidities. To address these issues, we developed an acellular nanofibrous scaffold with encapsulated growth factors designed to enhanced articular cartilage repair. Our goal is to evaluate this technology in vitro and pilot a large animal model for eventual translation into human subjects. METHODS/STUDY POPULATION: Hyaluronic acid (HA, $65 \mathrm{kDa}$ ) will be methacrylated ( $40 \%$ modification, MeHA) and conjugated with cell-adhesive (RGD) groups. A solution of $4 \% \mathrm{wt} / \mathrm{vol} \mathrm{MeHA}, 2 \% \mathrm{wt} / \mathrm{vol}$ polyethylene oxide $(900 \mathrm{kDa}), 0.05 \% \mathrm{wt} / \mathrm{vol}$ Irgacure 2959 , and $0.005 \% \mathrm{wt} / \mathrm{vol}$ stromal cellderived factor-I $\alpha$ (SDF-I $\alpha$ ) and/or transforming growth factor- $\beta 3$ (TGF- $\beta 3$ ) will be prepared in $\mathrm{ddH}_{2} \mathrm{O}$. The solution will be electrospun onto a rotating mandrel to achieve a dry scaffold thickness of $0.5 \mathrm{~mm}$. The scaffold matt will be UV cross-linked and $5 \mathrm{~mm}$-diameter samples will be cut out. Four groups of scaffolds will be prepared: MeHA, MeHA + SDF, MeHA + TGF, MeHA + SDF + TGF. All groups will be evaluated for fiber diameter, swell thickness, equilibrium compressive modulus, degradation rate, and growth factor release rate over 4 weeks $(n=10)$. Scaffolds will also be seeded with juvenile porcine MSCs $(5 \times 104)$ in $200 \mu \mathrm{L}$ of medium incubated for 24 hours. Seeded scaffolds will be evaluated for equilibrium compressive modulus, cell infiltration, and chondrogenesis at 4 and 8 weeks $(n=10)$. Scaffolds will then be evaluated in a juvenile Yucatan minipig cartilage defect model. In total, 6 animals will undergo bilateral knee surgery to create four $4 \mathrm{~mm}$-diameter fullthickness cartilage defects in each trochlear grove. All defects will receive microfracture to release marrow elements. Each knee will receive 2 scaffolds of the same group (replicates) with paired microfracture controls, resulting in a sample size of 3 . Animals will be sacrificed at 12 weeks and defects will be evaluated via non-destructive indentation testing for mechanical properties, microCT for defect fill and subchondral bone morphology, and histology for ICRS II Visual Histological Assessment Scoring. RESULTS/ANTICIPATED RESULTS: Our preliminary studies have shown reliable replication of electrospun MeHA scaffolds. We anticipate cross-linking density to correlate positively with compressive modulus, and negatively with swell thickness, degradation rate, and growth factor release rate. We anticipate the addition of SDF-I $\alpha$ and TGF- $\beta 3$ to increase cell infiltration and chondrogenesis, respectively, within seeded scaffolds. Similarly, we expect minipig defects treated with growth factor-releasing scaffolds to show greater mechanical properties, defect fill, and ICRS II score compared with MeHA scaffolds without growth factor. DISCUSSION/SIGNIFICANCE OF IMPACT: This study has the potential to show how an HA-based cell-free scaffold can be augmented with 2 growth factors that act synergistically to improve cartilage repair in a large animal model. This technology would improve upon the cellfree scaffolds already used clinically for autologous matrix-induced chondrogenesis and is directly translatable.

Age-related change in 5-HT6 receptor availability in healthy male volunteers measured with I ICGSK2 I 5083 PET

Rajiv Radhakrishnan, Nabeel Nabulsi, Edward Gaiser, Jean-

Dominique Gallezot, Shannan Henry, Beata Planeta, Shu-fei Lin, Jim Ropchan, Wendol Williams, Evan Morris, Deepak Cyril D'Souza, Yiyun Huang, Richard E. Carson and David Matuskey Yale School of Medicine, Yale University, New Haven, CT, USA

OBJECTIVES/SPECIFIC AIMS: The serotonin receptor $6(5-\mathrm{HT} 6)$ is a potential therapeutic target given its distribution in brain regions that are important in depression, anxiety, and cognition. This study sought to investigate the effects of age on 5-HT6 receptor availability using I IC GSK2 I5083, a PET ligand with affinity for 5-HT6 in the striatum and 5-HT2A in the cortex. METHODS/STUDY POPULATION: In total, 28 healthy male subjects (age range: 23-52 years) were scanned with IIC-GSK2I5083 on the HR + PET 
scanner. Time-activity curves in regions-of-interest were fitted with multilinear analysis-I method. Binding potentials (BPND) were calculated using cerebellum as the reference region and corrected for partial volume effects. RESULTS/ANTICIPATED RESULTS: In 5-HT6 rich areas, regional I IC-GSK2 I 5083 displayed a negative correlation between BPND and age in the caudate $(r=-0.4 \mathrm{I}, p=0.03)$ ( $14 \%$ change per decade), and putamen $(r=-0.30, p=0.04)$ ( $11 \%$ change per decade), but not in the ventral striatum and pallidum. Negative correlation with age was also seen in cortical regions $(r=-0.4 \mathrm{I}, p=0.03)$ (7\% change per decade), consistent with the literature on 5-HT2A availability. DISCUSSION/SIGNIFICANCE OF IMPACT: This is the first in vivo study in humans to examine the effect of age on 5-HT6 receptor availability. The study demonstrated a significant age-related decline in 5-HT6 availability (BPND) in the caudate and putamen.

\section{0}

Association between source case cavitation on chest radiograph and QuantiFERON-TB Gold In-Tube conversion among close contacts of active tuberculosis cases in Brazil

Lauren A. Saag, Marcelo Cordeiro-Santos', Afranio Kritski ${ }^{2}$, Bruno Andrade $^{3}$, Solange Cavalcante ${ }^{4}$, Betina Durovni ${ }^{4}$, Megan Turner ${ }^{5}$, Marina Figueiredo ${ }^{5}$, Valeria Rolla ${ }^{6}$ and Timothy Sterling ${ }^{5}$

' Fundação Medicina Tropical Doutor Heitor Vieira Dourado, Manaus, Brazil; ${ }^{2}$ Universidade Federal do Rio de Janeiro, Departamento de Clínica Médica, Rio de Janeiro, Brazil; ${ }^{3}$ Instituto Brasileiro para Investigação da Tuberculose, Bahia, Brazil; ${ }^{4}$ Secretaria Municipal de Saúde do Rio de Janeiro, Rio de Janeiro, Brazil; ${ }^{5}$ Division of Infectious Diseases, Department of Medicine, Vanderbilt University, Nashville, TN, USA; ${ }^{6}$ Instituto Nacional de Infectologia Evandro Chagas, Rio de Janeiro, Brazil

OBJECTIVES/SPECIFIC AIMS: QuantiFERON-TB Gold In-Tube (QFT) conversion from negative to positive, is regarded as a marker of recent latent tuberculosis infection and may be predictive of incident active tuberculosis (TB) disease. However, it remains unclear how conversion is influenced by individual and environmental factors, including the infectiousness of the source case to whom the contact was exposed. We aimed to examine the effect of infectiousness of TB in the source case, as measured by presence of cavitation on chest X-ray, on the incidence of QFT conversion among close contacts of the pulmonary TB index case, after adjusting for potential confounding by contact and source case characteristics. METHODS/STUDY POPULATION: The Regional Prospective Observational Research for Tuberculosis (RePORT)-Brazil is an ongoing prospective cohort study that enrolls close contacts of culture-confirmed pulmonary TB patients and follows them for 24 months for development of active TB. Demographic, clinical, and diagnostic information are obtained at baseline and during follow-up at clinical visits and by telephone. QFT testing is performed at baseline and repeated after 6 months if the baseline QFT is negative. A positive IFN- $\gamma$ value is defined as $>0.35 \mathrm{lU} / \mathrm{mL}$, as recommended by the manufacturer and the CDC, and QFT conversion is defined as a negative QFT at baseline followed by a positive QFT at 6 months. RESULTS/ANTICIPATED RESULTS: Among 260 enrolled contacts with nonpositive baseline QFT results and 6 months of follow-up, 198 (76\%) were retested with QFT 6 months after enrollment. Of those retested, 26 (13\%) converted to positive. Presence of any cavitation in the source case, based on chest radiography, was significantly associated with QFT-conversion (ORunadjusted =2.4, 95\% Cl: I.0-5.7). Additional univariate analyses revealed that QFT conversion was associated with black and brown race (compared with white race) of the contact, current smoking and current alcohol use in the source case. After adjusting for potential confounders (age, sex, and race of the contact and current smoking of the source case), the association between source case cavitation and QFT conversion remained (ORadjusted $=2.595 \% \mathrm{Cl}$ : I.0-6.2). As of December 6, 20I7, none of the QFT-retested contacts had developed active TB, with a median follow-up of 12.3 months (IQR: 7.I-13.I). We anticipate that ongoing enrollment and follow-up may yield cases of active TB; future analyses will provide greater precision for examining predictors of QFTconversion and its association with incident TB. DISCUSSION/SIGNIFICANCE OF IMPACT: Our preliminary results agree with published literature suggesting the infectiousness of TB in the index case is a predictor of incident LTBI. Along with recent LTBI, immune suppression, HIV co- infection, and type 2 diabetes are considered risk factors for progression to active TB disease. Because only a small proportion of persons progress from $\mathrm{LTBI}$ to active TB disease, it is not appropriate to treat all persons with LTBI. Thus, more research is needed to identify groups at highest risk for QFTconversion and incident $T B$ disease, so these groups can be targeted for $T B$ prevention, interventions, and facilitate a decline in TB incidence and mortality.

Associations between inflammatory markers and negative symptoms in individuals with schizophrenia: Converging evidence

David Goldsmith', Robert Cotes', Brian J. Miller², Michael T.

Treadway $^{3}$, Elaine F. Walker ${ }^{3}$ and Andrew H. Miller'

' Department of Psychiatry and Behavioral Sciences, Emory

University; ${ }^{2}$ Department of Psychiatry and Health Behavior, Augusta

University; ${ }^{3}$ Department of Psychology, Emory University

OBJECTIVES/SPECIFIC AIMS: Negative symptoms of schizophrenia, including motivational deficits, social withdrawal, poverty of speech, decreased emotional reactivity, and psychomotor retardation, have been shown to be most predictive of functional impairment and poor outcome in patients with schizophrenia. Furthermore, these symptoms tend not to be responsive to antipsychotic medications. Inflammation could be one mechanism underlying these difficult to treat symptoms. METHODS/STUDY POPULATION Three cohorts of patients, reflecting different phases of disease, were studied. One cohort was comprised of a sample of patients with deficit schizophrenia (characterized by primary and enduring negative symptoms; $n=17)$, nondeficit patients $(n=39)$, and healthy controls $(n=28)$. ANOVA and multivariate general linear models were used to compare groups, and linear regression models were used to examine relationships between inflammatory cytokines and negative symptoms. The second cohort was comprised of 80 individuals at clinical high risk for psychosis from the North American Prodromal Longitudinal Study. Linear regression models examined the relationship between baseline inflammatory markers and subsequent negative symptoms at follow-up visits up to 2 years. The third cohort consisted of patients with treatment-resistant schizophrenia (TRS) on clozapine $(n=10)$. Correlations were performed to examine relationships between inflammatory markers and negative symptoms. In a subgroup of patients from this third sample, resting state functional connectivity analyses were performed on fMRI data to explore relationships between inflammatory markers and connectivity in brain reward circuitry. RESULTS/ ANTICIPATED RESULTS: In a sample of patients with the deficit syndrome of schizophrenia $(n=17)$, a subtype of the disorder characterized by primary and enduring negative Symptoms, tumor necrosis factor (TNF) was significantly increased relative to nondeficit patients $(n=39)$ and healthy controls $\left(n=28 ; F_{2.57}=3.5 \mathrm{I}, p=0.036\right)$, and predicted total negative symptoms $(\beta=0.3 \mathrm{I}, p=0.012)$, alogia $(\beta=0.30, p=0.024)$, and blunted affect $(\beta=0.31, p=0.018)$ items of the Positive and Negative Symptom Scale in linear regression models while controlling for antipsychotics. In another sample of individuals at clinical-high risk for psychosis $(n=80)$, baseline concentrations of TNF significantly predicted negative symptoms, including anhedonia, apathy, and loss of interest in linear regression models, at the 6 month $(\beta=0.25, p=0.0 \mathrm{II})$ and I2-month follow-up $(\beta=0.39, p=0.00 \mathrm{I})$. Interleukin (IL)-I receptor antagonist also predicted these symptoms at the 6-month follow-up $(\beta=0.21, p=0.037)$. In a third sample $(n=10)$ of patients with TRS treated with clozapine, IL-I $\beta$ was correlated with passive/ apathetic social withdrawal $(r=0.657, p=0.039)$ and disturbance of volition $(r=0.686, p=0.029)$ items of the Positive and Negative Symptom Scale and the global avolition-apathy scores of the Scale for the Assessment of Negative Symptoms $(r=0.75 \mathrm{I}, p=0.012)$. Finally, in a small subsample $(n=5)$ of patients from this TRS cohort for whom we collected fMRI data, we found resting-state functional connectivity from a right nucleus accumbens seed to a cluster in medial prefrontal cortex. We found relationships between higher inflammation and decreased connectivity for TNF $(r=-0.64)$ and CRP $(r=-0.89)$. DISCUSSION/SIGNIFICANCE OF IMPACT: Taken together, these preliminary data show the predicted relationship between inflammatory markers and negative symptoms and demonstrate the reproducibility of TNF and other monocytic-derived cytokines as reliably elevated in schizophrenia and associated with negative symptoms across samples of patients with schizophrenia and individuals at high risk for psychosis. Cytokines may exert their effects via their impact on brain reward circuitry, and could represent novel treatment targets for motivational deficits and negative symptoms of schizophrenia. 